\title{
A Set of Computer Aided Automatic Control Experiments for Undergraduate Students
}

\author{
YUSUF BUĞDAY, MEHMET ÖNDER EFE \\ Department of Electrical and Electronics Engineering, TOBB University of Economics and Technology, Ankara, Turkey
}

Received 22 October 2009; accepted 24 May 2010

\begin{abstract}
In a typical control systems course, students are expected to develop the notion of open loop and closed loop first. Under the feedback, traditional control theory offers many elegant solutions to control problems yet teaching those concepts experimentally is an art. This article describes how the notion of feedback control could be developed by utilizing a computer aided experimental setup available at the laboratories of TOBB University of Economics and Technology. A DC motor control system is considered and step by step development of the understanding of feedback control is explained. Didactic aspect is taken care of throughout the manuscript and extensions to research are emphasized. (c) 2010 Wiley Periodicals, Inc. Comput Appl Eng Educ 21: 300-312, 2013; View this article online at wileyonlinelibrary.com/journal/cae; DOI 10.1002/cae.20472
\end{abstract}

Keywords: control experiments; undergraduate control education

\section{INTRODUCTION}

Use of computers and rapid increase in their speed and capabilities with affordable prices has been a strong influence on the engineering education which have been blended with the computer-based tools and approaches. Availability of several simulation software and development platforms have changed the teaching of engineering issues dramatically and today, the students, having all necessary tools installed on their computers, have the capability of prototyping the ideas instantly. Furthermore, most institutions are now covered by wireless networks enabling the students to access every kind of supplementary material. With such an amazingly technology, computer, web, and communication centric environment, class materials as well as the teaching methods need continuous update. The field of automatic control is one of such areas that influenced significantly from the possibilities offered by such advancements. Despite computer-based classroom teaching is one side of the whole issue; the other side is the exploitation of computer centric technologies in laboratory experiments that encompasses the concepts of telelab, virtual lab, or web-based interactive lab. This article focuses on the laboratory side with the goals of conveying a comprehensive understanding of feedback, control, simulation, dynamics, frequency response, and key techniques in the closed loop controller design such as PID, pole placement, fuzzy control, and sliding mode control (SMC).

In the past, many works have been reported to describe the alternative methods utilizing computer aid in automatic control

Correspondence to M. Ö. Efe (onderefe@etu.edu.tr). CC 2010 Wiley Periodicals, Inc. experimentation. Duan et al. [1] consider a web enabled control apparatus, an inverted pendulum, enabling the identification, prototyping, and refinement remotely. Another work reporting internet-based experimentation is by Casini et al. [2], where it is emphasized that the proposed system does not require being in the lab. The students perform the experiments remotely with having the full fledge of using Matlab/Simulink environment. Kamış et al. [3] present a motor control system developed for PID controller prototyping and typical transient responses and frequency response pictures are given. Huba and Simunek focus on teaching PID control techniques via the WebLab described in Ref. [4], where the system level architecture and the security of the web system is highlighted together with the possibilities of implementing various models and controllers at different complexities. Another work emphasizing the virtual instrumentation is due to Shahri [5], enabling web-based experimentation of Lead and Lag compensators. Wagner [6] stipulates 10 pragmatic design rules for the development of computer-assisted learning systems, and in Ref. [7] and overview of control education for several countries are elaborated.

Although web-based learning or remote learning is one avenue, this article presents a computer-centric prototyping approach requiring the student's presence at the lab. Tested implementations of the approaches adopted at the Department of Electrical and Electronics Engineering of TOBB ETU are presented. The article is organized as follows. The second section describes three simple experiments and simulations. The third section describes the DC motor control setup, and the experiments designed on it are defined in the fourth section. The fifth section is devoted to the question of how this viewpoint can be connected 
to the viewpoint of a researcher. The structuring of the lab experiments and the concluding remarks are given at the end of the article.

\section{SIMPLE EXPERIMENTS TO UNDERSTAND THE NOTION OF FEEDBACK}

This section describes several experiments where the computer use is restricted to simulation environments. The motivation of having such a work in the lab work.

\section{On-Off Control}

Perhaps the simplest approach to describe feedback is based on on-off technique. In the laboratory, students are asked to build the resistor-capacitor system shown in Figure 1 and to figure out how on-off scheme could be implemented utilizing a single off-theshelf operational amplifier (opamp). Most students remember the nonlinear saturating nature of opamp taught in the circuit analysis course and realize the feedback system should realize

$$
u(t)=\left\{\begin{array}{cc}
V_{\mathrm{cc}} & r(t)-y(t)>0 \\
-V_{\mathrm{cc}} & r(t)-y(t)<0
\end{array}\right.
$$

where $V_{\mathrm{cc}}$ is the supply voltage of the opamp and $r(t)$ is the target output. Once completed, the student comprehends the concept of state, feedback, and on-off type control. The output of the first order system is forced toward a desired voltage and since all quantities are in voltages, it is easier for a student to attribute meaning to measured signals. This experiment is a quick start experiment available in the lab work of control systems course.

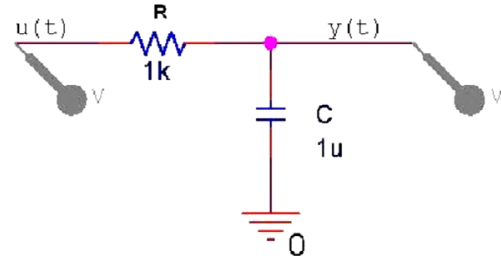

Figure 1 Pspice drawing of the system to be controlled. [Color figure can be viewed in the online issue, which is available at wileyonlinelibrary.com.]

\section{Control of a Second Order System: A Simulation Example}

In this part, students are asked to determine the components of a feedback control system depicted in Pspice environment. The given design is shown in Figure 2 and the plant is composed of a first order system followed by an integrator, which is described by (2).

$$
\ddot{y}=-\dot{y}+u
$$

Decomposing the blocks yields the dynamics above as well as the controller that is composed of a proportional plus derivative action. The difference computer yields the error $e=r-y$, and the PD controller computes a control signal given by

$$
u=\dot{e}+5 e
$$

After understanding the role of each block, students realize the same system in Matlab/Simulink environment and compare the results for $r(t)=1$ with zero initial conditions and $t \leq 5 \mathrm{~s}$. The goal

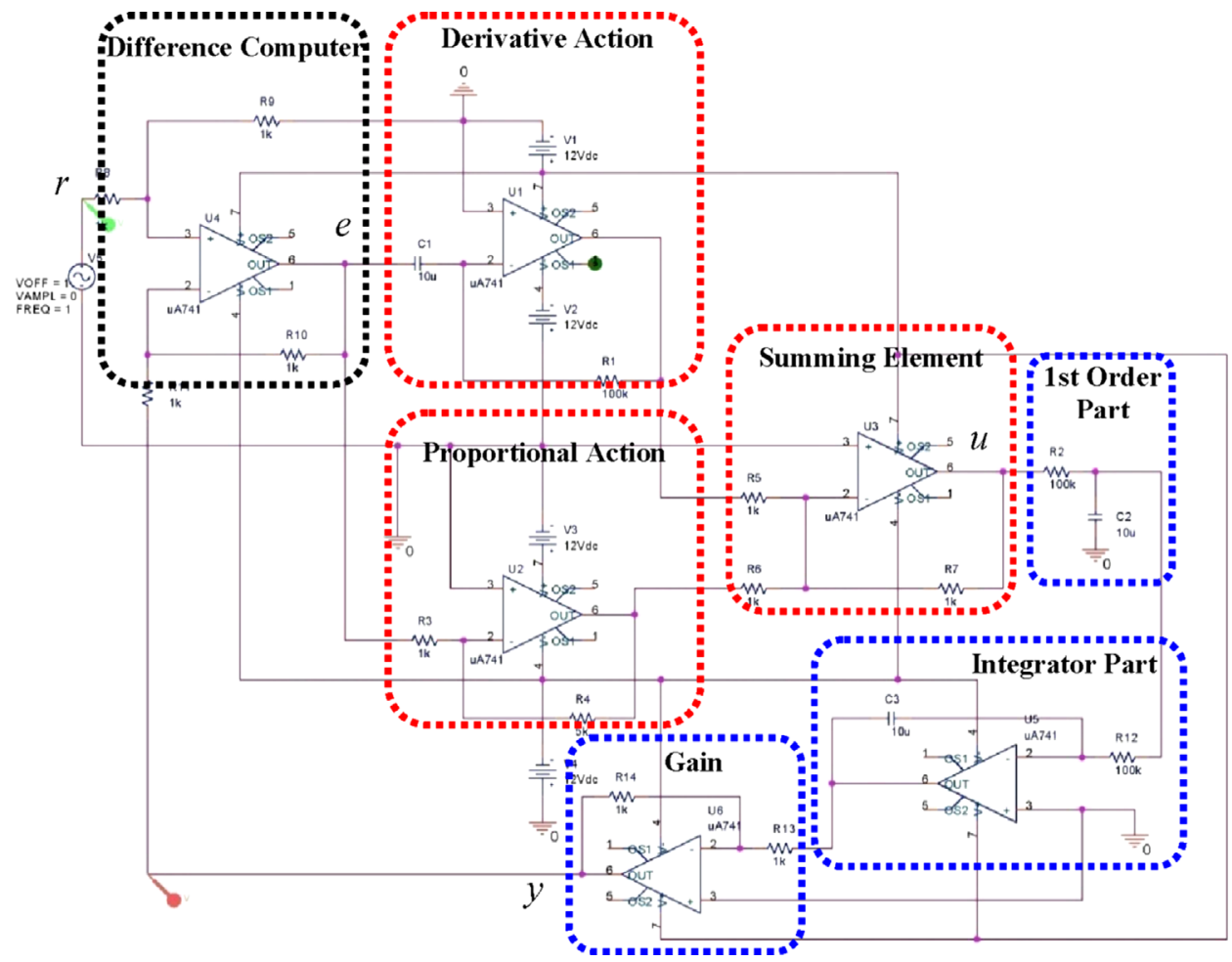

Figure 2 Pspice drawing of the closed loop control system. [Color figure can be viewed in the online issue, which is available at wileyonlinelibrary.com.] 
(a)

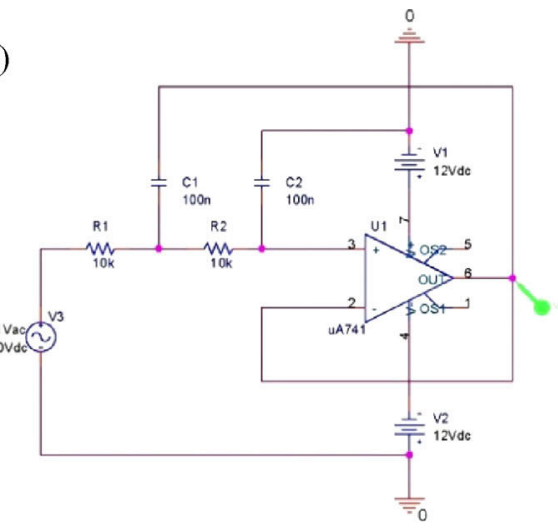

(b)
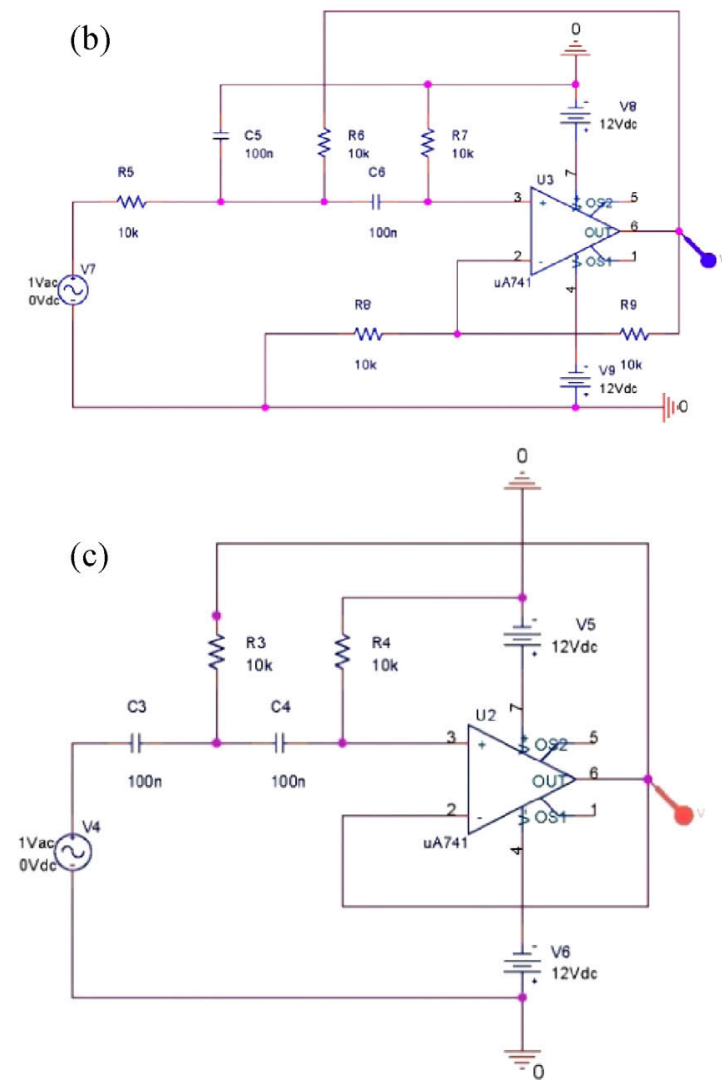

Figure 3 (a) Lowpass filter, (b) bandpass filter, and (c) highpass filter. [Color figure can be viewed in the online issue, which is available at wileyonlinelibrary.com.]

of such an experiment is to familiarize students with simulation tools like Matlab and Pspice, and to describe overshoot, rise time, peak time, and settling time concepts according to the obtained results.

\section{Understanding the Frequency Domain}

Teaching the frequency domain is one of the critical issues in almost all engineering disciplines. The method we follow at this part of the article is to describe three Sallen-Key filters designed for lowpass, bandpass, and highpass behaviors. Students build the circuits in Pspice environment, shown in Figure 3, and simulate for (a)

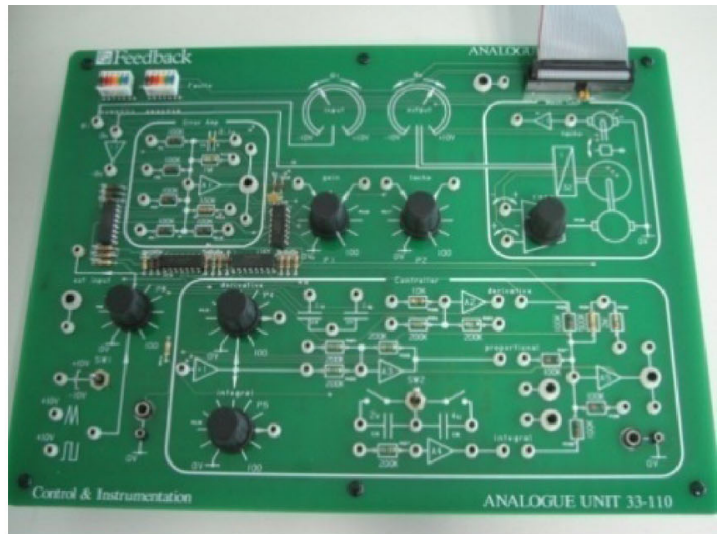

(b)

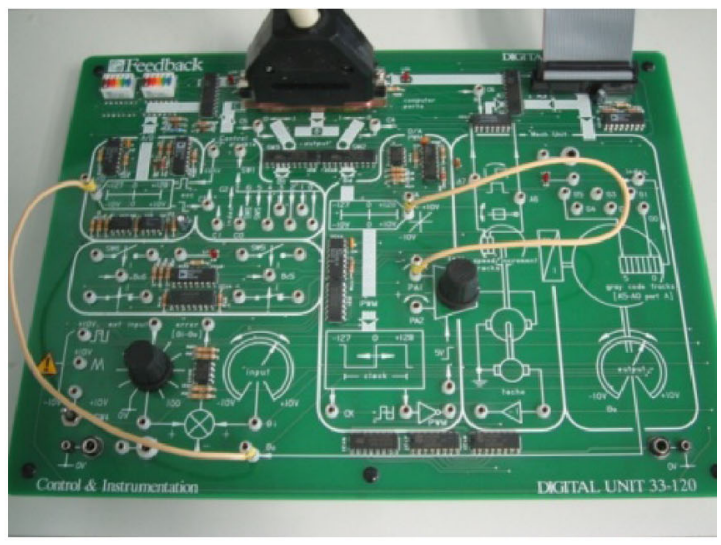

(c)

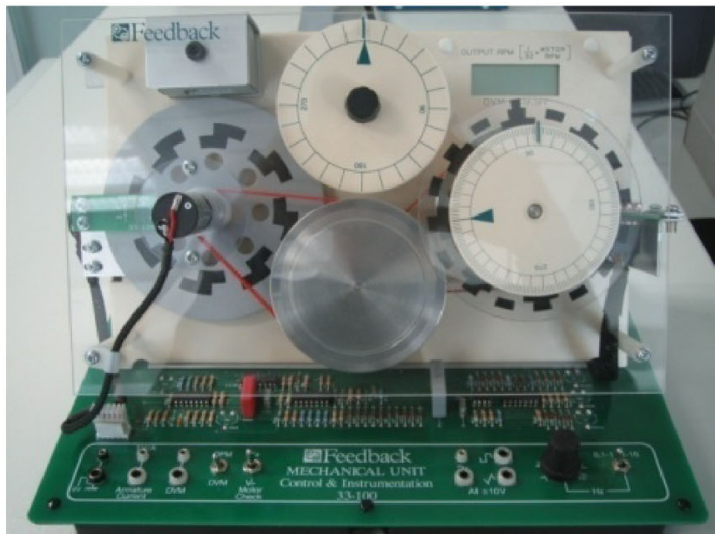

Figure 4 (a) Analog control unit, (b) digital control unit, and (c) mechanical unit. [Color figure can be viewed in the online issue, which is available at wileyonlinelibrary.com.]

sinusoidal inputs of unity magnitude with logarithmic frequency values. The results are recorded, and then the approximate Bode magnitude plots are constructed. The aim of such a simulation study is to gain an insight about justifying the theoretical results via simulation.

\section{MOTOR CONTROL SETUP}

The motor control setup utilized in this work is composed of a host computer, analog control unit shown in Figure 4a, the digital 


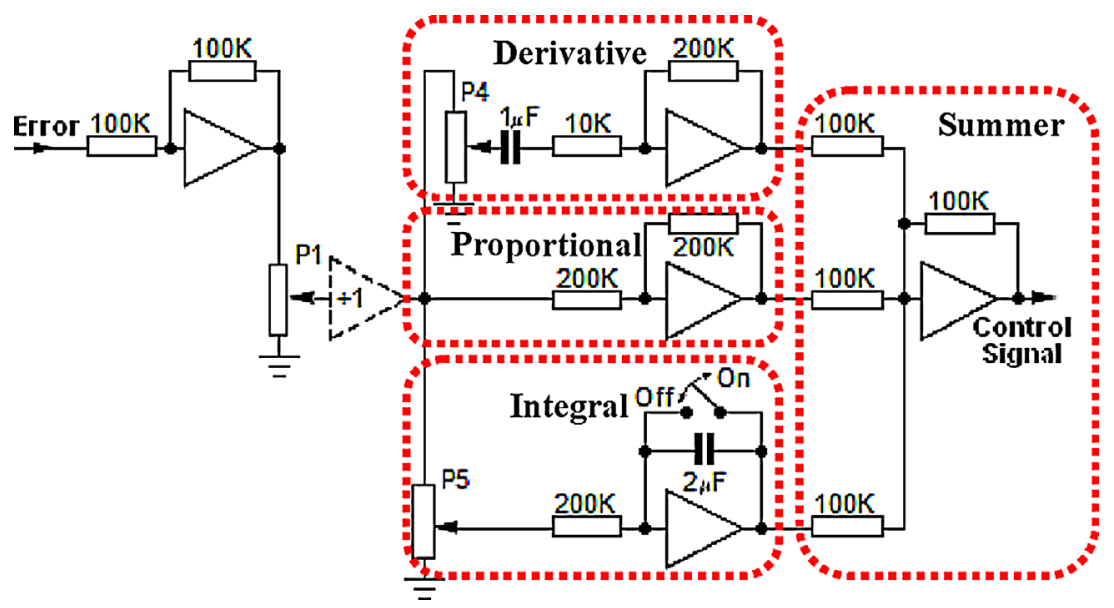

Figure 5 PID controller to be realized. [Color figure can be viewed in the online issue, which is available at wileyonlinelibrary.com.]

control unit, shown in Figure $4 \mathrm{~b}$ and a mechanical unit as shown in Figure 4c. An I/O card is installed into the host computer and this card functions in providing the handshaking between the digital control unit and the software platform, run by the host computer. The analog unit provides experimenting proportional, integral, and derivative (PID) type controllers and variants, while the digital control unit enables implementing user-defined control laws on Matlab/Simulink. The computer-assisted facility therefore provides a platform to develop novel control algorithms as well as the conventional ones both for teaching and for research purposes.

Mechanical unit is equipped with a tachogenerator, analog, and digital encoders. The unit possesses a power amplifier to drive the installed DC motor and few standard reference signals are generated on-board for possible trajectory tracking experiments. Practically, the introduced development platform is ideal for junior level students to develop a feeling of feedback control, further, limited sampling rate and limited control voltage makes it a good test bed for those pursuing research oriented solutions. The next section describes how such an experimental facility could be used in teaching the fundamental concepts of automatic control.

\section{LINEAR CONTROL EXPERIMENTS UTILIZING DC MOTOR CONTROL HARDWARE}

This section describes the teaching approach based on the DC motor control setup. The methodology contains analog and digital control examples demonstrating the concept progressively.

\section{PID Control via Analog Control Unit}

Due to their practical nature, well-explained dynamical response and widespread preferability in industry, the most common technique in the practice of automatic control systems is the PID controllers and their variants. In the laboratory, we aim to demonstrate PID controller implementation on the DC motor control setup with analog unit shown in Figure 4a. The PID controller structure is shown in Figure 5, where it is possible to implement many combinations of proportional, integral, and derivative effects and to see their implications on the system response. Further, the available setup enables to reset the integrator manually. The phys- ical connectivity is shown in Figure 6, which is simple enough to realize during a lab session.

After designing the controller and building the closed loop system on the analog unit, there are a number of steps that have to be followed as a procedure by the students with manually applying a step function as the input signal to the system. These steps consist of the trials of some variations for the coefficients of proportional, integral, and derivative actions. An exemplar input and output graph of the closed loop system is obtained on the oscilloscope as given in Figure 7, where the PID coefficients are adjusted to the default settings provided by the manufacturer.

The students are asked to vary the coefficient of the proportional action for several times while keeping the coefficients of integral and derivative actions zero. They are directed to apply step function of magnitude $10 \mathrm{~V}$ for each parameter configuration and observe the motor position physically on the mechanical unit as well as on the oscilloscope. In the second step, integral and derivative actions are added to proportional control and the effect of integral action is investigated by varying the coefficient and by changing the value of the capacitor in the feedback path of operational amplifier, while the proportional and derivative coefficients are constant. A similar process is repeated in the third step that investigates the effect of derivative action while proportional and integral coefficients are nonzero constants. Thus, the effects of the proportional, integral, and derivative actions on steady-state error, oscillations, rise time, and settling time of system response are separately studied. In this manner, students gain an insight about one of the PID control scheme and practical considerations in its practice.

\section{Pole Placement Technique via Digital Control Unit}

Pole placement method, a well-known approach of the control theory, is based on the idea that the poles of a closed loop system may be placed at any desired location by means of state feedback through an appropriate state feedback gain matrix under the assumption that the dynamic model of the system is known and controllability matrix has full rank. In this experiment the students are given the dynamic model of the system as given in (1) and (2).

The model of the DC servo motor system, located at the mechanical unit mentioned before, is defined while neglecting the nonlinear characteristic added to system by the strap mechanism 


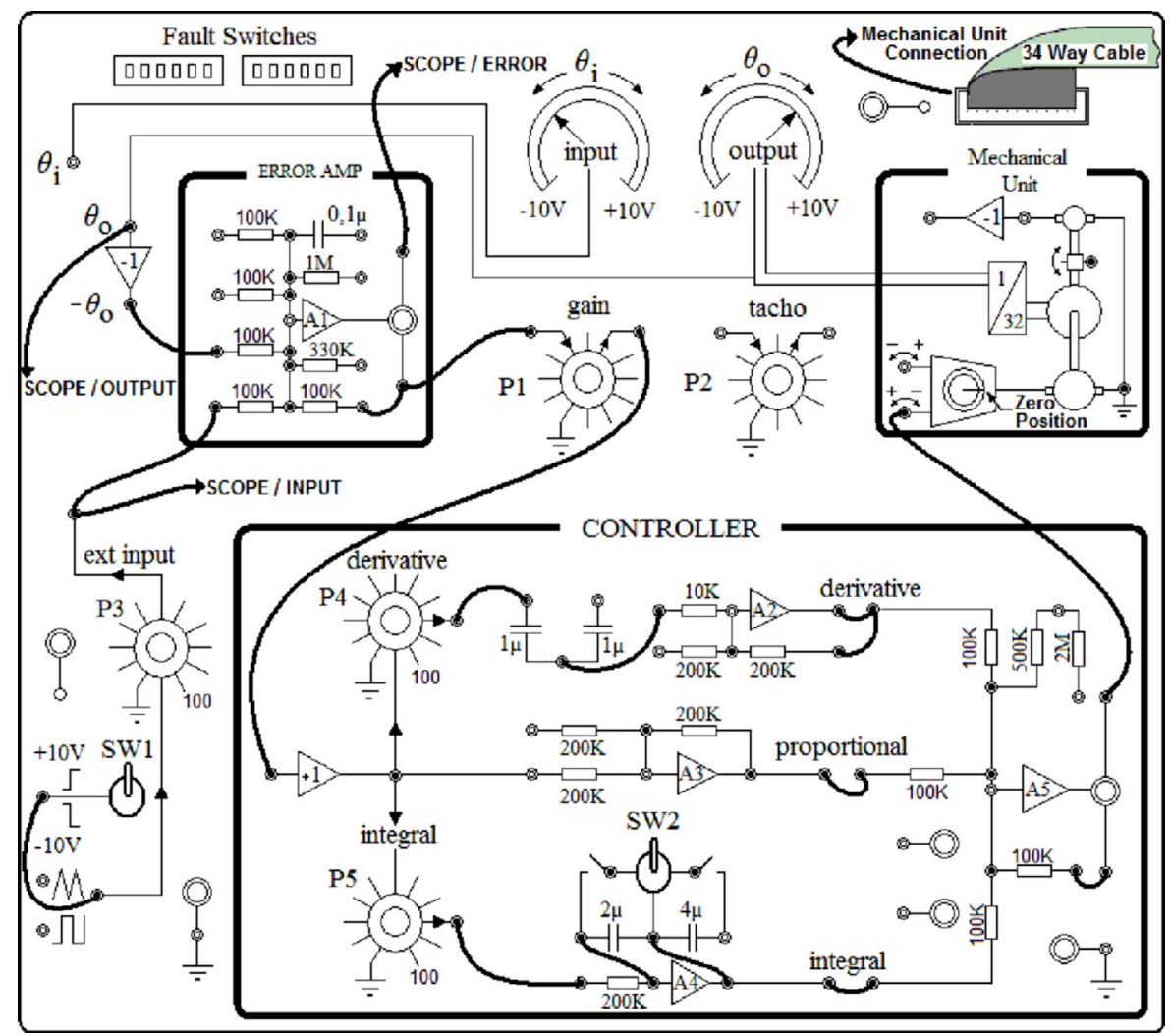

Figure 6 Physical connectivity for the desired PID controller on analog unit.

between the wheels. State space model of single input single output (SISO) linear time invariant system can be considered as common expressions

$$
\begin{gathered}
\dot{x}(t)=A x(t)+B u(t) \\
y(t)=C x(t)
\end{gathered}
$$

where $x$ is state vector containing the angular position and angular velocity, $y$ is the output signal (angle), $u$ is control signal (voltage) and $A=\left[\begin{array}{cc}0 & 1 \\ 0 & -4.76\end{array}\right], B=\left[\begin{array}{l}0 \\ 3.19\end{array}\right], C=\left[\begin{array}{ll}1 & 0\end{array}\right]$ are constant a-valued matrices, respectively. The control signal $u(t)$ is defined

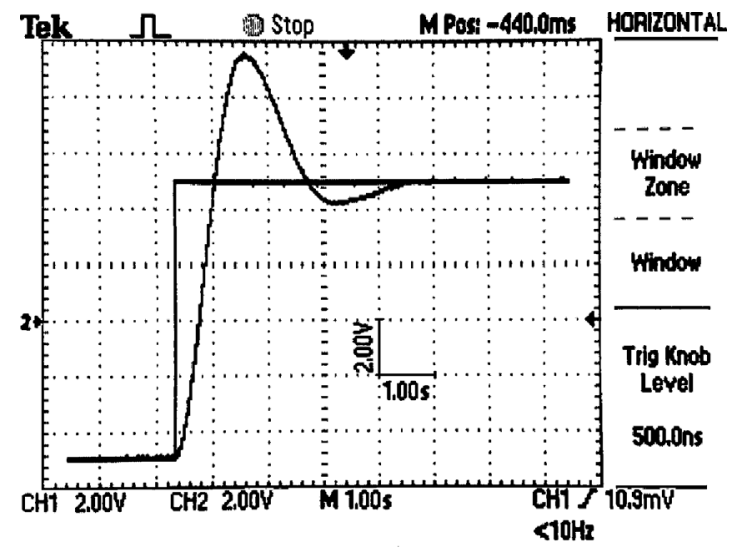

Figure 7 The response of the system to a step input. as below

$$
u(t)=-K x(t)+\operatorname{pr}(t)
$$

where $r(t)$ represents the reference signal and $K$ is the state feedback gain matrix of dimensions $1 \times 2$ and $p$ is a constant the is chosen to make steady-state error zero. Angular velocity is obtained from numerical derivative of the measured motor position. With this $A$ and $B$, the system is full state controllable, thus the poles of closed loop system can be mapped to any desired location by choosing a proper gain matrix $K$. The task given to students is to map the closed loop system poles to $s_{1}=1.8, s_{2}=1.9$, which were chosen arbitrarily. Further, while choosing the pole values, physical limitations of the system have to be considered in order to obtain a physically realizable control signal and system response. Students are instructed to obtain the state feedback gain matrix $K$ in Matlab by utilizing "place" command which computes the gain matrix by using the pair $A, B$, and the desired poles given above. Following this procedure, the students obtain the gain matrices for two different pairs of desired poles. After the calculation step ends, students realize the controller in Simulink environment, shown in Figure 8 . The students are advised to smooth the angular speed data as it is severely noisy. An exemplar realization is illustrated in Figure 8.

The angular position data of system, observed from the mechanical unit via the digital control unit, and the control signal, driving the DC motor via digital control unit, are both available as blocks in Simulink environment. A reference signal, which is a sine wave during the first $40 \mathrm{~s}$ and a square wave during the next $40 \mathrm{~s}$, is chosen. This particular choice of the reference signal 


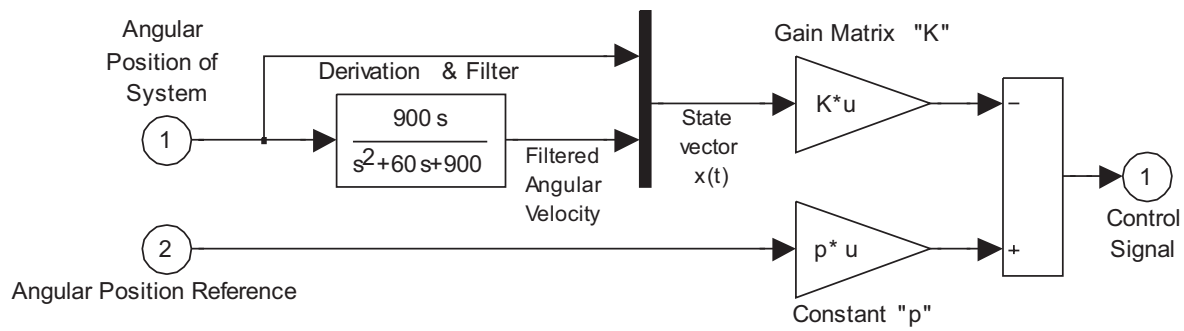

Figure 8 Simulink implementation of state feedback with approximate angular speed measurements.

enables to see the performance when the command is smooth and differentiable and when it displays abrupt changes. For the chosen reference signal and the closed loop poles, the response of the system is shown in Figure 9. As seen in the figure, the system can follow the reference signal, while the tracking error varies in an acceptable range.

The aim of this experiment is to introduce the principles of state feedback using pole placement technique via a computer aided platform providing fast prototyping possibility. The importance of selecting proper closed loop poles and filtering the numerical derivation of data are the two main issues understood by students through this experiment.

\section{Observer Design via Digital Control Unit}

State feedback is a powerful technique in automatic control systems if all state variables are known. However, not all state variables are available in practice and for this reason; unknown
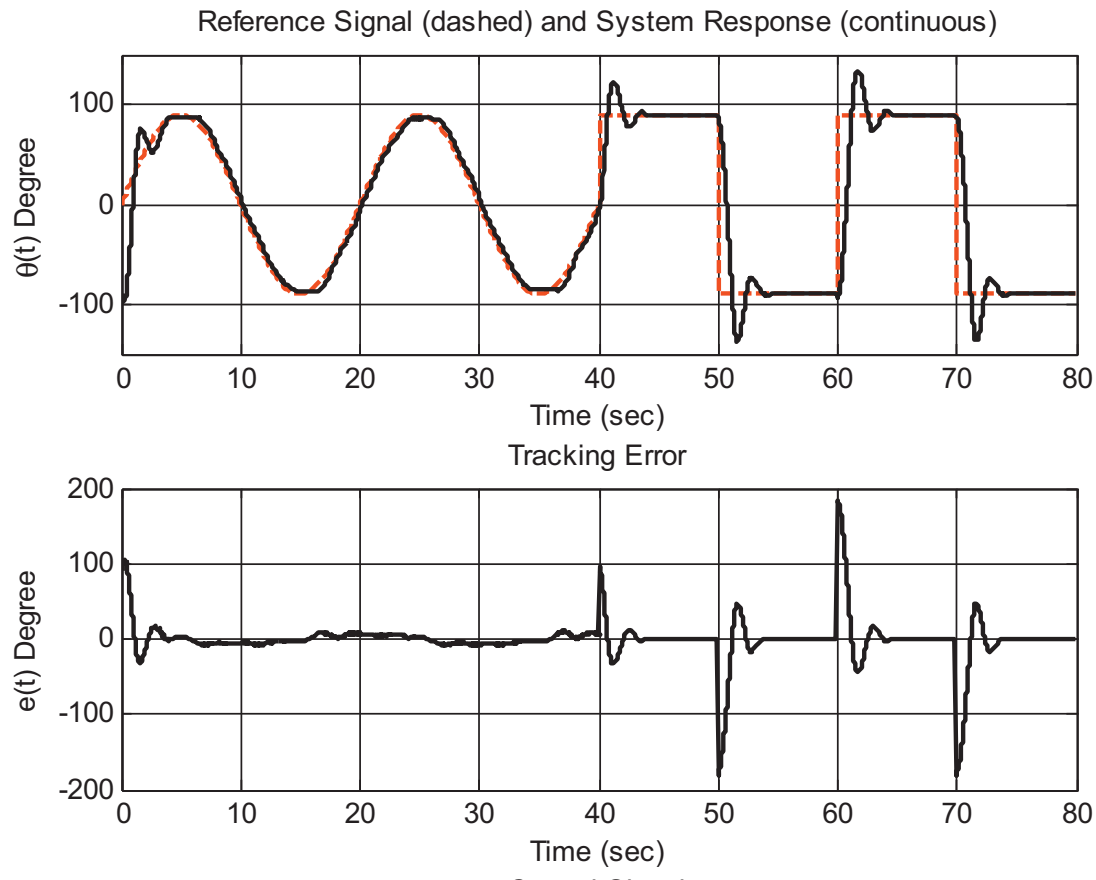

Control Signal

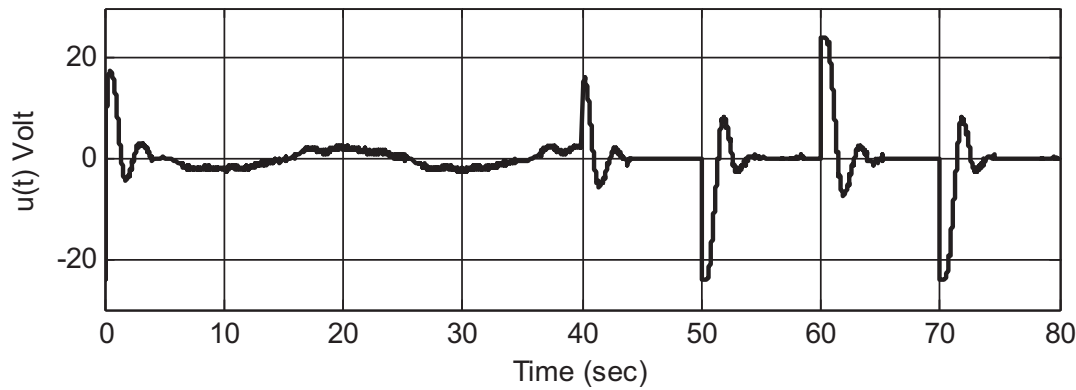

Figure 9 System response to the reference signal (top), tracking error (middle), and control signal (bottom) results for the closed loop system poles are $s_{1}=1.8, s_{2}=1.9$, and $P$ is 1.057. [Color figure can be viewed in the online issue, which is available at wileyonlinelibrary.com.] 


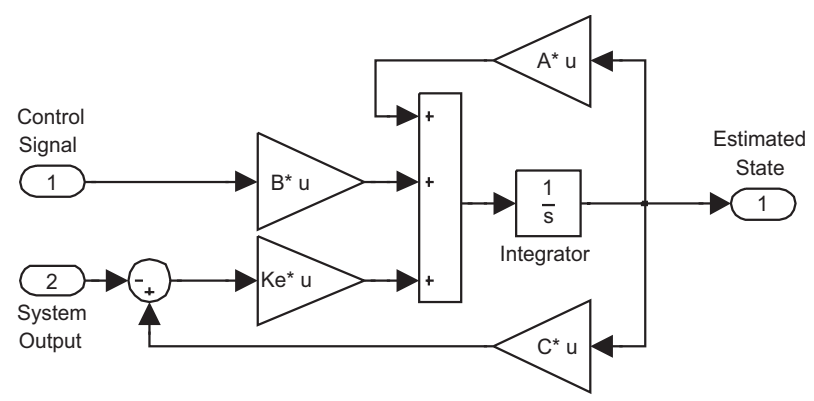

Figure 10 Simulink model of the observer designed in experiment.

state variables must be estimated by observers to be able to perform the state feedback. In this sense, students are expected to obtain the state variables of the servo system by observers in this experiment. The mathematical model of the observer is defined as given in ()

$$
\dot{\hat{x}}=A \hat{x}+B u+K_{e}(y-C \hat{x})
$$

where $\hat{x}$ is the estimated state and $K_{e}$ is the observer gain matrix intensifying the error between the measured angular position of the system $y$ and the estimated angular position $C \hat{x}$ to improve the correctness of the estimation. The students are asked to calculate the observer gain matrix $K_{e}$, given as

$$
K_{e}=\left[\begin{array}{ll}
k_{e_{1}} & k_{e_{2}}
\end{array}\right]^{\mathrm{T}}
$$

Defining the error as $e:=x-\hat{x}$ it is possible to derive the tracking error dynamics.

$$
\dot{e}=\left(A-K_{e} C\right) e
$$

Claiming a desired pair of eigenvalues for the matrix $A-K_{e} C$ enables us to determine $k_{e_{1}}$ and $k_{e_{2}}$. The observer is realized in MATLAB/Simulink environment as shown in Figure 10. The external control of the system is maintained by a PID controller while the observer predicts the system states.

By this way, students can easily apply various $K_{e}$ gain matrices, calculated for different possible observer poles, to system and experience the effects of the chose values of poles. Furthermore, estimated and measured states of the system are compared and performance of the observer is displayed in Figures 11 and 12 where the desired eigenvalues of $A-K_{e} C$ (the poles of the observer) are $s_{1,2}=-1$ for the first case and $s_{1,2}=-8$ for the second case. It is seen from the figures that the students comprehend the varying speed of convergence for different $K_{e}$ selections.

Through the experiment, extraction of the missing state variables of a process is conveyed to the students. In this case, position is measurable yet the angular speed is not. The observer provides a prediction for the angular speed. According to the results for the two selections, the students learn the observer is unable to catch if its poles are not adequately away from the origin (see Fig. 11), if
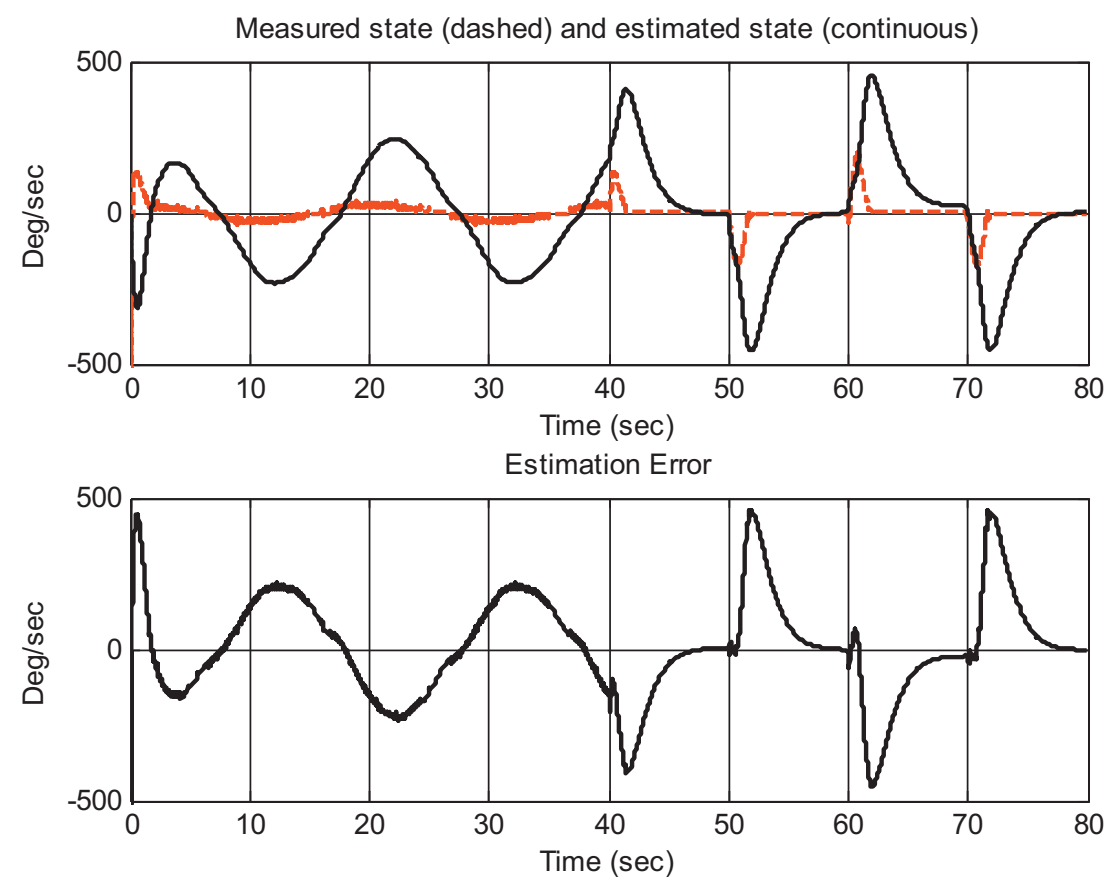

Figure 11 Motor speed estimation results for $s_{1,2}=-1$. [Color figure can be viewed in the online issue, which is available at wileyonlinelibrary.com.] 

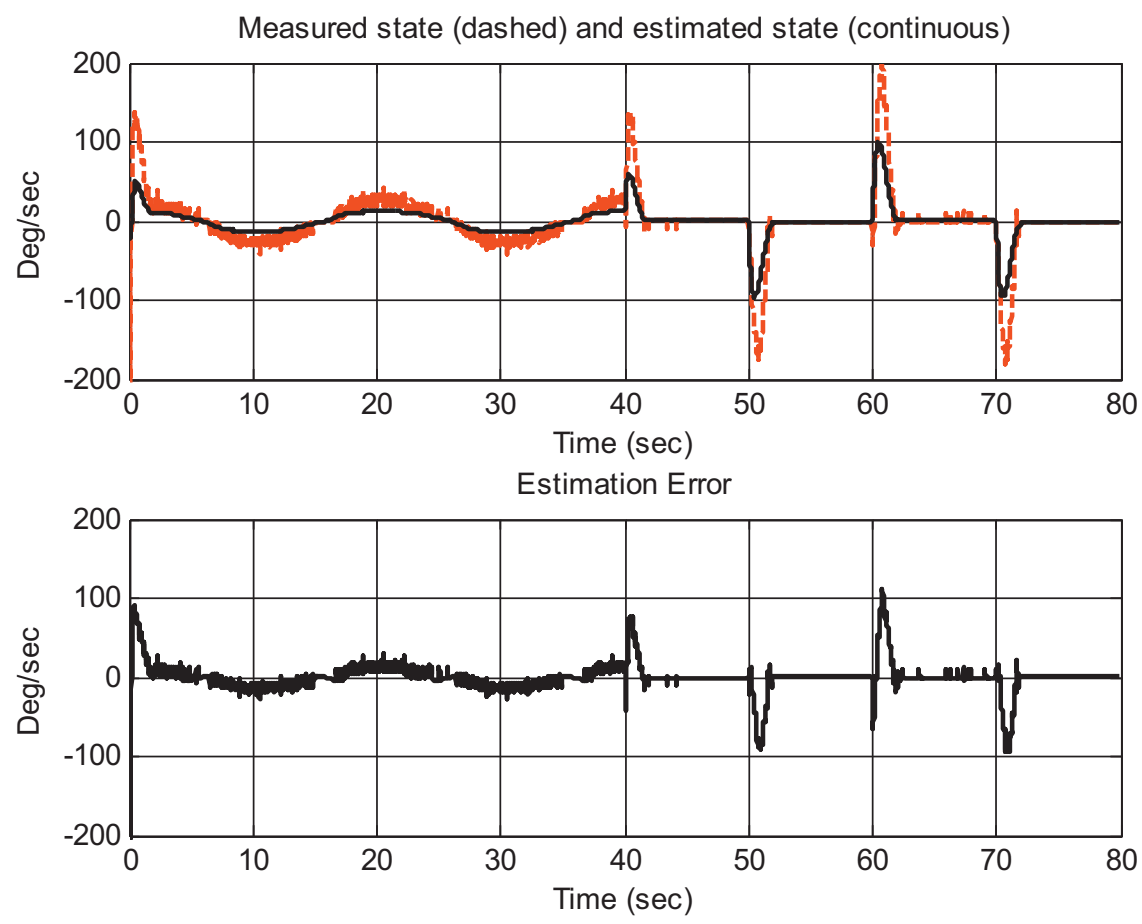

Figure 12 Motor speed estimation results of for $s_{1,2}=-8$. [Color figure can be viewed in the online issue, which is available at wileyonlinelibrary.com.]

they are chosen appropriately, the observer removes the spurious content in the angular speed and produces a much smoother speed data as shown in the top row of Figure 12.

\section{NONLINEAR CONTROL EXPERIMENTS}

This section dwells on how the concepts of linear control can be extended to nonlinear control laws while maintaining stability and performance expectations. Two popular approaches are experimented, namely, SMC and fuzzy control, which are explained in the sequel.

\section{Sliding Mode Control via Digital Control Unit}

SMC is one of the most popular nonlinear control schemes used frequently in the literature. The philosophy of the design based on the guidance of the error vector toward a predefined subspace of the phase space, called the sliding manifold, and to maintain it there. The design of this subspace, on the other hand, is a particularly selected one, such that once the error vector gets trapped to it, the motion thereafter takes place in the vicinity of this subspace and the error (and its derivatives) converge the origin [8-10].

Referring to the state space model described in (4) and (5), we define the errors as $e_{1}=x_{1}-r_{1}$ is the positional error, $e_{2}=x_{2}-r_{2}$, is the error in angular velocity with $r_{2}=\dot{r}_{1}$ and $r_{2}$ is differentiable too. Define the switching variable as

$$
\sigma=e_{2}+\lambda e_{1}=\left(\frac{d}{\mathrm{~d} t}+\lambda\right) e_{1}
$$

where the positive valued parameter $\lambda$ determines the slope of the line in the space spanned by $e_{1}$ and $e_{2}$. After taking the time derivative of (10) and exploiting (4) and (5) lets us have the control input in the expression as given below

$$
\begin{gathered}
\dot{\sigma}=\dot{e}_{2}+\lambda \dot{e}_{1} \\
=\dot{x}_{2}-\dot{r}_{2}+\lambda e_{2} \\
=-4.76 x_{2}+3.19 u-\dot{r}_{2}+\lambda e_{2}
\end{gathered}
$$

Clearly for a $Q>0$, a reaching dynamics given by $\dot{\sigma}=$ $-Q \operatorname{sgn}(\sigma)$ would force any initial value of $\sigma$ to zero. Equating (11) to $-Q \operatorname{sgn}(\sigma)$ and solving for the control signal lets us have

$$
u=\frac{1}{3.19}\left(4.76 x_{2}+\dot{r}_{2}-\lambda e_{2}-Q \operatorname{sgn}(\sigma)\right)
$$

The control law above ensures $\sigma=0$ in finite time, further, it introduces a certain level of robustness against modeling uncertainties such as the adverse effect of the belt used in this system. In the practice, the sign function is replaced by the function in (13) to avoid the chattering, a phenomenon that arises due to the sign measurement of a quantity that is close to zero.

$$
\operatorname{sgn}(z) \cong \frac{z}{|z|+\delta}, \quad \delta>0
$$

In this experiment, the effects of the following on the system response are investigated.

- Reaching law parameter Q.

- The slope parameter $\lambda$.

- The sign function smoothing parameter $\delta$.

The sliding mode controller in (12) is realized in the Simulink environment with the aforementioned approximate angular velocity information. Students are expected to vary the three parameters mentioned above, thus they analyze the characteristics and qualities of the system response as well as the control signal peculiar to each specific case. 

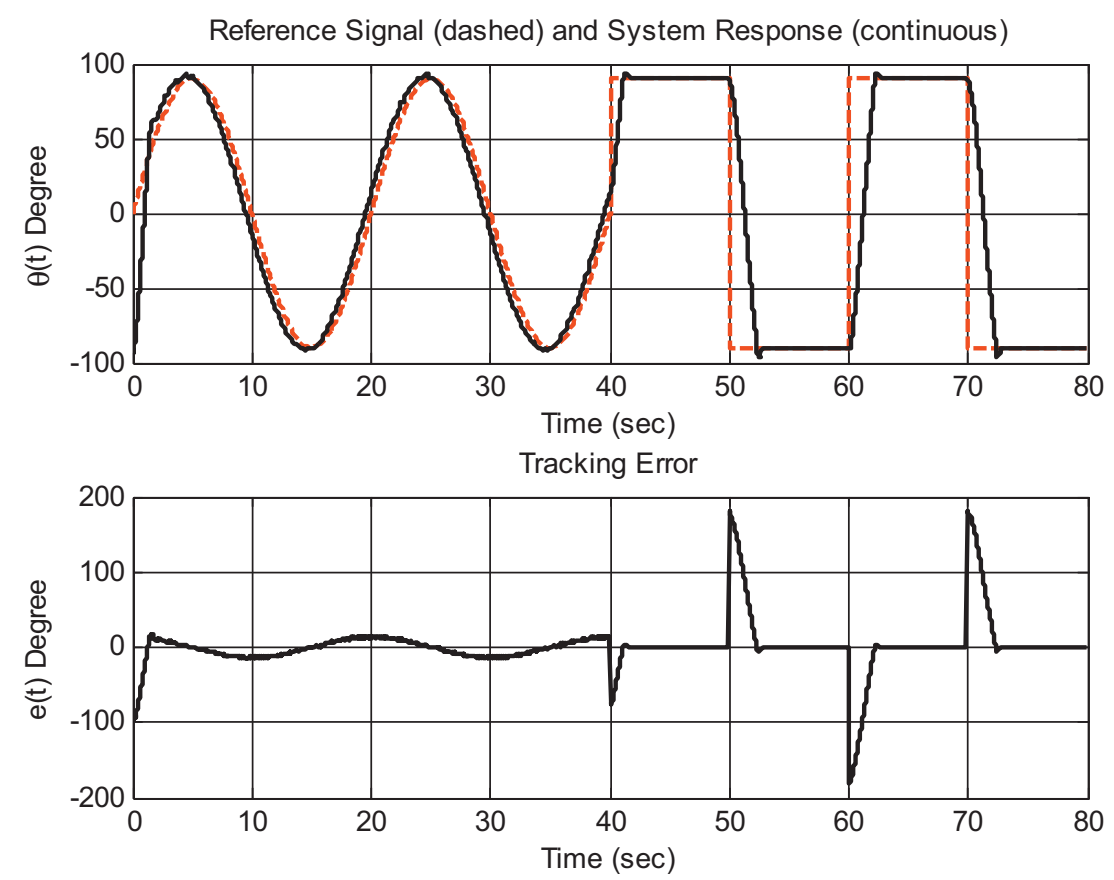

Control Signal

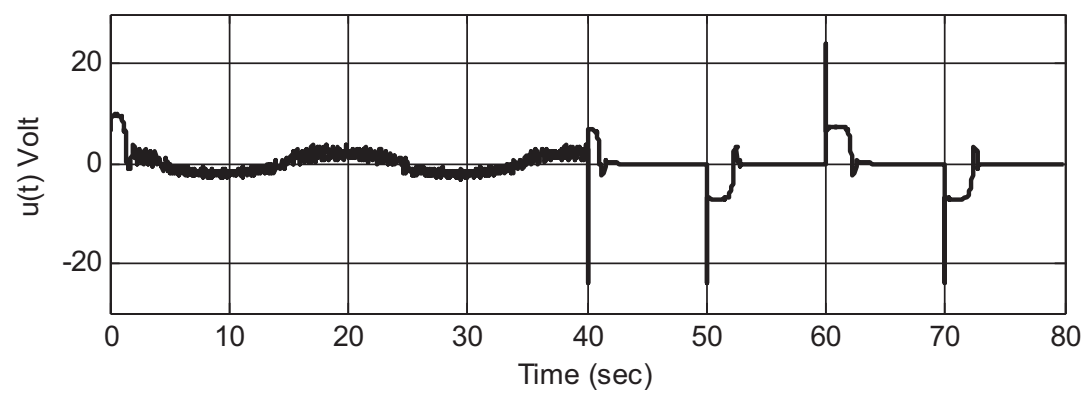

Figure 13 System response to the reference signal (top), tracking error (middle), and control signal generated by the sliding mode control (bottom). [Color figure can be viewed in the online issue, which is available at wileyonlinelibrary.com.]

Initially, the values of $\lambda, \delta$, and $Q$ are selected as $2,0.8$, and 10 , respectively. Thus, the system response follows the desired reference signal with an admissible level of error as seen in Figure 13. When the reference signal suddenly changes, the controller produces a high amplitude signal locally in order to force the value of $\sigma$ toward zero. For safety reasons, the control signal is within the range $\pm 24 \mathrm{~V}$ and when there are abrupt changes, the control signal is likely to reach (or saturate at) these hard bounds.

The phase space behavior of error is illustrated as subplots in Figure 14. The top left subplot depicts the reaching phase of the experiment, where we see that the error vector hits the sliding line and remains in the vicinity of it. In the top right subplot, the students see the sliding mode, which guide the error vector toward the origin of the phase space. After the $40 \mathrm{~s}$ of run, square wave reference signal becomes active, the controller is still able to stabilize the system yet it is difficult to see the sliding regime as clear as in the sinusoidal reference case.

Three parameters, namely $\lambda, \delta$, and $Q$, characterizing the response of the controlled system, are varied on a one-at-a-time basis. Increasing the value of reaching law parameter $Q$ provides faster reaching, more robustness but causes more chattering.
Increasing the value of slope parameter $\lambda$ provides faster convergence during the sliding mode. Increasing the value of sign function smoothing parameter $\delta$ worsens robustness; on the other hand decreasing $\delta$ magnifies the chattering. The students acquire how a robust control law is implemented and the dependence of performance on the design parameters is understood.

\section{Fuzzy Control via the Digital Control Unit}

Fuzzy control is another nonlinear control experiment available in the description of the laboratory. The philosophy that lies behind is based on the descriptions in terms of linguistic quantities. The way to describe these quantities is to define membership functions characterizing sets whose boundaries are unsharp, that is, fuzzy, $[X]$. Fuzzy control is an active research field and the goal of the experiment is to teach the basics of fuzzy control as well as establishing links in between the undergraduate level control education and its research extensions. The first step of the experiment is to understand the fuzzy quantification of a variable called $e$. In Figure 15 , three fuzzy sets are depicted which were asked once for every new value of $e$, which changes in time. 
Phase Space for Sinusodial Reference

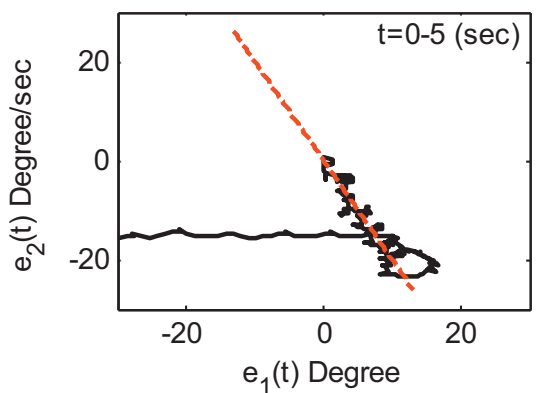

Phase Space for Square Reference

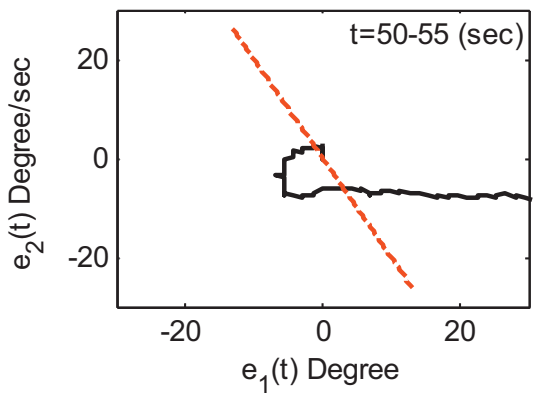

Phase Space for Sinusodial Reference

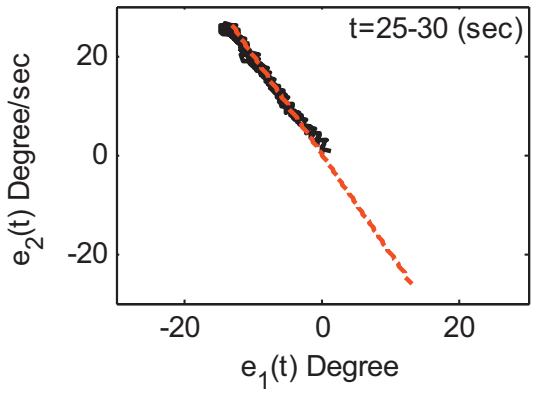

Phase Space for Square Reference

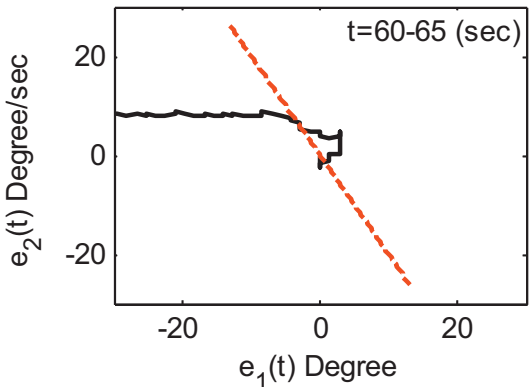

Figure 14 The trajectories of the error in the phase space for different periods of an experiment. [Color figure can be viewed in the online issue, which is available at wileyonlinelibrary.com.]

If a different process is to be activated according to different regional values of $e$, fuzzy logic is the best alternative to partition the input space $\mathfrak{R}$, to which $e$ belongs, and activates dedicated processes whenever the necessary premises are held to a good extent. More explicitly, for two input and single output fuzzy system, the rules of the form.

$$
I F e_{1} \in P_{i} \text { and } e_{2} \in W_{i} T H E N u=y_{i}
$$

determine the local conclusion and the fuzzy system interpolates in between many such local conclusions to build a global map. In the above rule structure, $P_{i}$ and $W_{i}$ denote the fuzzy sets corresponding to some linguistic label, positive, negative, or zero (see Fig. 15).
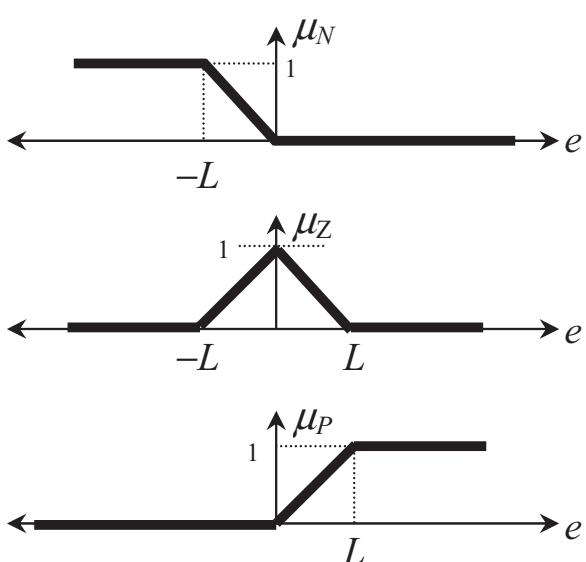

Figure 15 Membership functions, negative $(\mathrm{N})$ is on the top, zero $(\mathrm{Z})$ is in the middle, and positive $(\mathrm{P})$ is at the bottom.
In this experiment, a standard Sugeno type fuzzy controller is used and defined by $R=9$ rules with triangular membership functions as shown above. Position error $\left(e_{1}\right)$ and its derivative $\left(e_{2}\right)$ are applied as inputs to fuzzy controller. Input and output relation is given as in (14).

$$
u=\frac{\sum_{i=1}^{R} y_{i} \prod_{j=1}^{m} \mu_{i j}\left(e_{j}\right)}{\sum_{i=1}^{R} \prod_{j=1}^{m} \mu_{i j}\left(e_{j}\right)}
$$

Membership functions, seen in (14) and depicted in Figure 15 are described explicitly as

$$
\begin{gathered}
\mu_{\mathrm{N}}(e)=\max \left(\min \left(-\frac{e}{L}, 1\right), 0\right) \\
\mu_{\mathrm{Z}}(e)=\max \left(\min \left(1+\frac{e}{L}, 1-\frac{e}{L}\right), 0\right) \\
\mu_{\mathrm{P}}(e)=\max \left(\min \left(\frac{e}{L}, 1\right), 0\right)
\end{gathered}
$$

The membership functions with a design parameter $L$ are implemented in Simulink environment and the overall fuzzy controller in (14) is then realized for experimentation. The teaching goal here is to convey the effect of different $L$ and $y_{i}(i=1,2, \ldots$, $R$ ) selections on the performance. The latter describes the defuzzifier, which converts the fuzzy quantities to crisp values. Initially the students are given the value of $L$ quantifying the positional error as $33^{\circ}$ and that quantifying the error in the angular speed as $82^{\circ} / \mathrm{s}$. Likewise, the an initial selection for the defuzzifier parameters $(y)$ is as given below (Fig. 16)

$$
y=\left[\begin{array}{lllllllll}
-22 & -16 & -8 & -2 & 0 & 2 & 8 & 16 & 22
\end{array}\right]^{\mathrm{T}}
$$



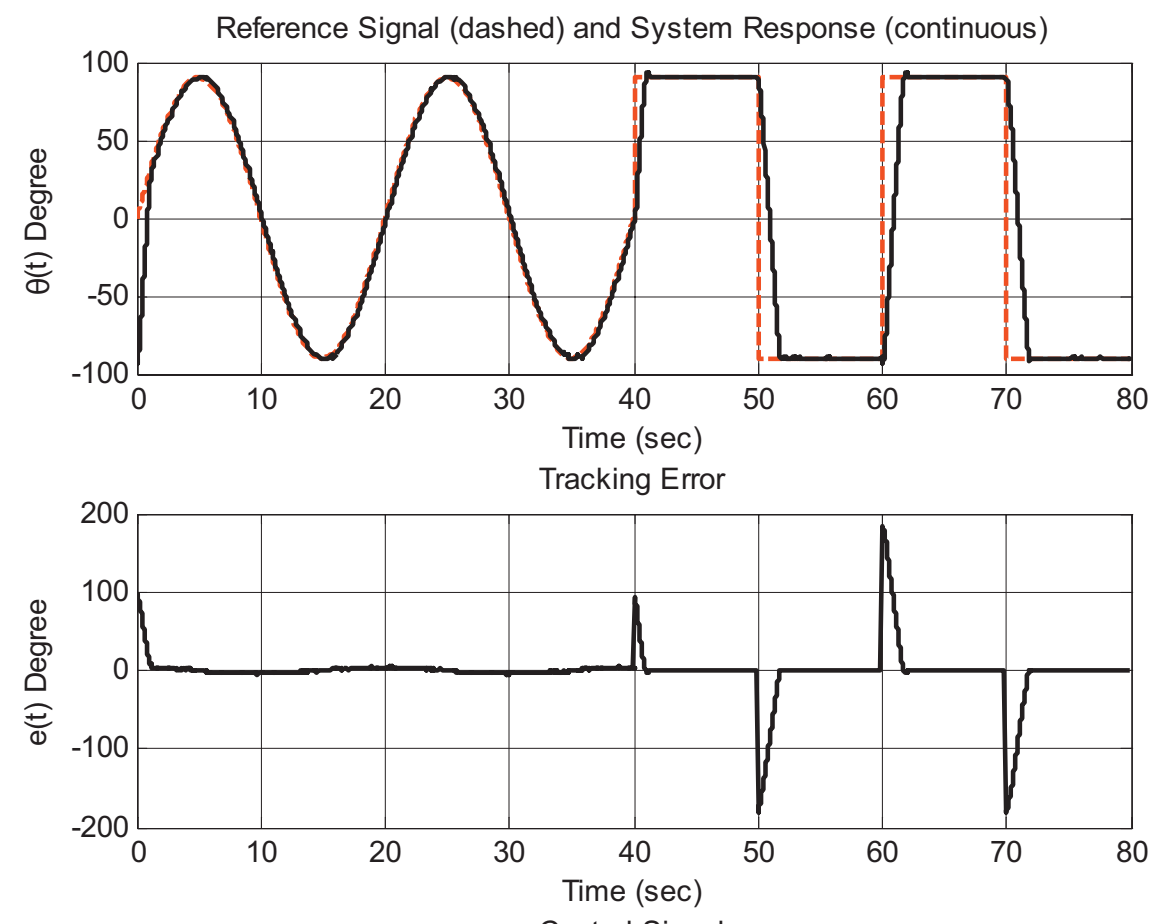

Control Signal

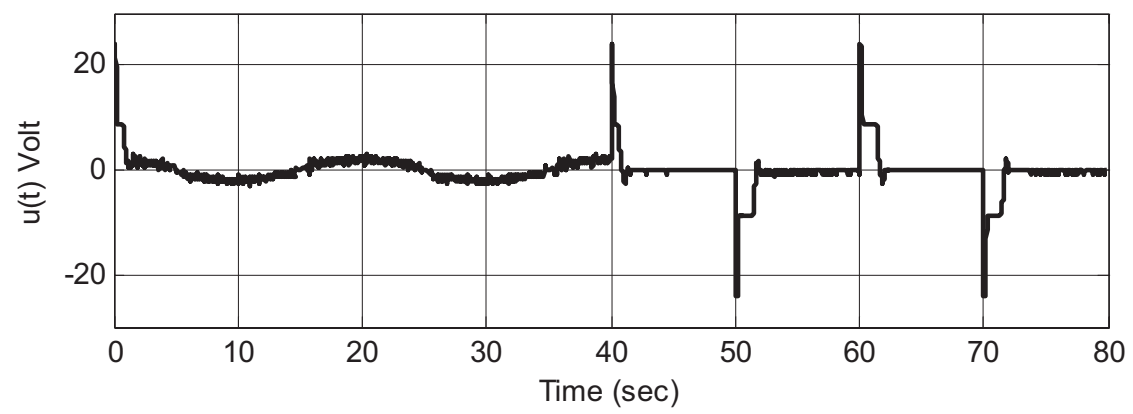

Figure 16 System response to the reference signal (top), tracking error (middle), and control signal generated by fuzzy controller (bottom). [Color figure can be viewed in the online issue, which is available at wileyonlinelibrary.com.]

Defined control surface and obtained results are shown in Figure 17. The closed loop performance of the fuzzy controller is similar to sliding mode controller, however, the transient response of the system has shorter rising and settling times while using fuzzy controller by choosing proper values for $y_{i}$, providing faster converging. Besides, the steady-state performance of the fuzzy controller is sufficiently good.

The aim of the experiment is to teach how feedback control can be accomplished without knowing the system dynamics. Since the student perform several experiments before trying the fuzzy control approach, they get a solid understanding of the DC motor dynamics and they become capable of writing the rules to stabilize the plant at a desired setpoint or around a desired trajectory as shown in Figure 14.

\section{STRUCTURING THE LABORATORY EXPERIMENTS}

Most engineering institutions offer the first control course in the third year of the undergraduate education. This course typically encompasses the topics from the ordinary linear differential equations to preliminary concepts of state space analysis. The courses

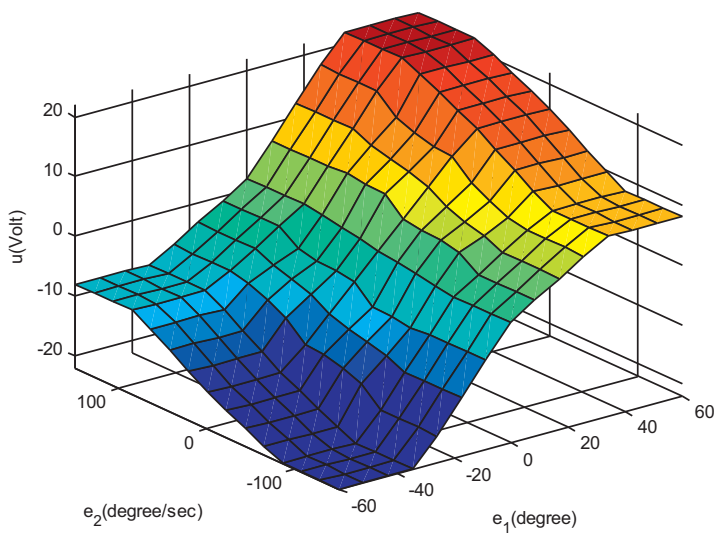

Figure 17 Control surface with the fuzzy system in (14). [Color figure can be viewed in the online issue, which is available at wileyonlinelibrary.com.] 
Table 1 Implementation Scheme for Junior and Senior Level Students

\begin{tabular}{lll}
\hline Experiment name & 3rd class students & 4th class students \\
\hline On-off control & Experiment \#1 & Prelab study \#1 \\
Control of a second order system: A simulation example & Experiment \#2 & Prelab study \#2 \\
Understanding the frequency domain & Experiment \#3 & Prelab study \#3 \\
PID control via analog control unit & Experiment \#4 & Experiment \#5 \\
Pole placement technique via digital control unit & Experiment \#6 (optional) \\
Observer design via digital control unit & Not included & Experiment \#2 \\
Sliding mode control via digital control unit & Not included & Experiment \#4 \\
Fuzzy control via digital control unit & & Experiment \#5 \\
\hline
\end{tabular}

at the senior level are more diverse containing the state space analysis, robust and nonlinear control techniques in depth. This article describes a set of laboratory experiments that is adaptable to both 3rd and 4th year students as detailed in Table 1. The assessment of the laboratory grade is based on the student's performance during the experiment as well as the report submitted till the next lab session.

Regarding the learning of the course material, students' fundamental gain is to get a solid understanding of the concept of feedback. Further, during the course, the students perform several simulations, where components behave ideally, and they grasp the notion of imprecision during the real-time experiments. The quality of the feedback signal directly influences the closed loop performance and this is understood as a critical parameter influencing the performance of different control schemes. Another important gain of the students is to develop a an engineering feeling, that is, complicated control laws could yield better precision yet simpler ones like the PID scheme are easy to implement with cheap hardware. Last but not the least, after completing the described set of experiments, the students have a clear understanding of the frequency domain and the frequency response of dynamic systems. Overall, the students experience the key notions of the control systems through the described experiments.

\section{CONCLUSIONS}

This article describes several experiments that familiarize the control engineering students with the use of simulation tools as well as real-time design and implementation issues. The novelty introduced here is the method followed. To emphasize once more, the use of simple strategies to convey the fundamental ideas of feedback control is possible via simulation tools yet to understand better, several experiments are described to strengthen the feeling of feedback and closed loop control expectations. The considered experimental setup is a good test bed for junior level students taking the automatic control course as well as for the students who aim at solving research problems and prototype nonlinear control laws on a physical system having limited computational capabilities. The article also provides how the experiments can be grouped to address 3rd and 4th class engineering students separately.

\section{ACKNOWLEDGMENTS}

This work was supported by TÜBITAK contract no 107E137.

\section{REFERENCES}

[1] B. Duan, K.-V. Ling, H. Mir, M. Hosseini, and R. K. L. Gay, An online laboratory framework for control engineering courses, Int J Eng Educ 21 (2005), 1068-1075.

[2] M. Casini, D. Prattichizzo, and A. Vicino, The automatic control telelab: A user friendly interface for distance learning, IEEE Trans Educ 46 (2003), 252-257.

[3] Z. Kamış E. E. Topcu, and I. Yüksel, Computer aided automatic control education with a real-time development system, Comput Appl Eng Educ 13 (2005), 181-191.

[4] M. Huba and M. Simunek, Modular approach to teaching PID control, IEEE Trans Ind Electron 54 (2007), 3112-3121.

[5] A. M. Shahri, Advanced Control E-Laboratory (ACeL) Based on Virtual Instrumentation, IEEE CCECE 2003 Canadian Conference on Electrical and Computer Engineering, Montreal, Canada, Vol. 1, pp. 1-4, May 4-7, 2003.

[6] B. Wagner, From Computer Based Teaching to Virtual Laboratories in Automatic Control, 29th ASEE/IEEE Frontiers in Education Conference, San Juan, Puerto Rico, November 10-13, 1999.

[7] IEEE Control Systems Magazine, April 1996.

[8] J. Y. Hung, W. Gao, and J. C. Hung, Variable structure control: A survey, IEEE Trans Ind Electron 40 (1993), 2-22.

[9] K. D. Young, V. I. Utkin, and Ü. Özgüner, A Control Engineer's Guide to sliding mode control, IEEE Trans Control Syst Technol 7 (1999), 328-342.

[10] X. Yu and O. Kaynak, Sliding-mode control with soft computing: A survey, IEEE Trans Ind Electron 56 (2009), 3275-3285.

\section{BIOGRAPHIES}

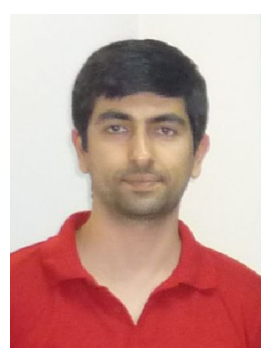

Yusuf Bugday received the B.Sc. Degree from the Department of Electrical and Electronics Engineering, TOBB University of Economics and Technology (Turkey) in 2004. He is now a Masters student at the same department. Mr. Bugday is a research assistant since 2008 and his research interests include the real time applications of control systems, fuzzy logic and intelligent systems.

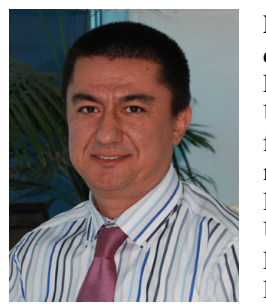

Prof. Mehmet Önder Efe received the B.Sc. degree from Electronics and Communications Engineering Department, Istanbul Technical University (Turkey) in 1993, and M.S. degree from Systems and Control Engineering Department, Bogaziçi University (Turkey), in 1996. He completed his Ph.D. study in Bogaziçi University, Electrical and Electronics Engineering Department in June 2000. Between August 1996 December 2000, he was with Bogaziçi University, Mechatronics Research and Application Center as a research assistant. During 2001, Prof. Efe was a postdoctoral research fellow at Carnegie 
Mellon University, Electrical and Computer Engineering Department, and he was a member of the Advanced Mechatronis Laboratory group. Between January 2002 and July 2003 he was with The Ohio State University, Electrical Engineering Department as a postdoctoral research associate. He worked at the Collaborative Center of Control Science. As of September 2003, he started working at Atilim University, Department of Mechatronics Engineering as an Assistant Professor. Prof. Efe was entitled Associate Professor in April 2004 and Full Professor in July 2009.

In August 2004, he has joined Electrical and Electronics Engineering Department of TOBB University of Economics and Technology. Prof. Efe was the head of the department between August 2004-July 2007 and between June 2008 to date. He has taken several administrative positions at TOBB ETU. Prof. Efe was the head of IEEE CSS Turkey Chapter between January 2007 and December 2008, and he is currently a Senior Member of IEEE. He serves as an Associate Editor for the journals IEEE Transactions on Industrial Electronics, Transactions of the Institute of Measurement and Control, International Journal of Industrial Electronics and Control and Advances in Fuzzy Systems. Prof. Efe is the author/co-author of more than 100 technical publications focusing on the applications of computational intelligence and systems \& control theory. Prof. Efe is married to Almula and he is the father of Nildeniz and Zeynep Burtay. 